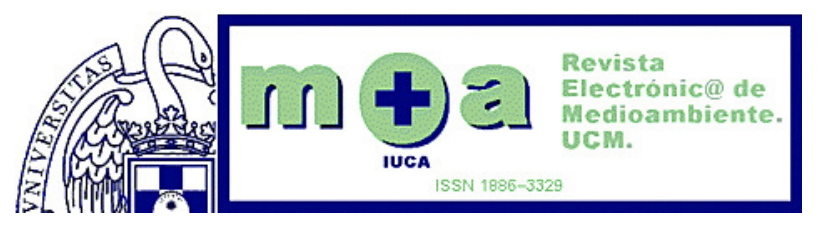

\title{
REPERCUSIÓN DE LAS AYUDAS AGROAMBIENTALES A \\ LA PRODUCCION INTEGRADA EN EL PROGRAMA DE DESARROLLO RURAL DE LA REGIÓN DE MURCIA
}

\author{
M. L. TUDELA SERRANO \\ Profesora Titular de Análisis Geográfico Regional. de la Universidad de Murcia \\ mltudela@um.es
}

M.M. RIQUELME NAVARRO

Licenciada en Ciencias Ambientales. Universidad de Murcia cabecicos@gmail.com

Recibido: 09-03-2012

Aceptado: $15-06-2012$

\section{RESUMEN}

La Producción Integrada es un sistema agrícola de obtención de vegetales que utiliza al máximo los recursos y los mecanismos de producción naturales, garantizando el desarrollo sostenible de las zonas rurales e incentivando una gestión de las tierras compatible con la necesidad de preservar el medio ambiente y el paisaje natural. La superficie de Producción Integrada en la Región de Murcia ha experimentado un considerable aumento a través de las ayudas recibidas en sus dos líneas de ayuda, siendo el almendro y el viñedo los cultivos con mayor superficie concedida, seguida de frutales y uva de mesa. Las ayudas agroambientales han tenido una importante aceptación en el sector agrario murciano, si bien no han cubierto las expectativas de un importante número de agricultores que han visto desestimadas sus solicitudes por falta de presupuesto.

Palabras clave: producción integrada, desarrollo rural, ayudas agroambientales.

Study environmental impact of the aid to agriculture in the integrated production rural development programme in the Region of Murcia

\section{ABSTRACT}

The Integrated Production is an agricultural system of obtaining vegetable that uses to the maximum the resources and the natural mechanisms of production, ensuring 
the sustainable development of the rural zones and stimulating a management of the compatible lands with the need to preserve the environment and the natural landscape. The surface of Production Integrated to the Region of Murcia has had a considerable increase across the helps received in his two lines of help, being the almondtree and the vineyard the cultures with major surface granted, followed of fruit trees and grape of table. The helps to the environmental agriculture have had an important acceptance in the agrarian sector, though they have not covered the expectations of an important number of farmers who have seen his requests misestimated for lack of budget.

Keywords: integrated production, rural development, environmental, agriculture.

\section{La repercussion des aides agricoles a la production intégrée dans le programme de développement rural de la Region de Murcia}

\section{RÉSUMÉ}

La Production Intégrée est un système agricole d'obtention de végétaux qui utilise au maximum les recours et les mécanismes naturels de production, en garantissant le développement durable des zones rurales et en stimulant une gestion des terres compatible avec la nécessité de préserver l'environnement et le paysage naturel. La surface de Production Intégrée dans la Région a expérimenté une augmentation considérable avec les aides reçues dans ses deux lignes d'aide, en étant l'amandier et le vignoble les cultures avec une plus grande surface accordée, suivie de fruitiers et un raisin de table. Les aides agraires ont eu une acceptation importante dans le secteur agraire, bien qu'ils n'ont pas couvert les attentes d'un nombre important d'agriculteurs qui ont vu mésestimées ses demandes par manque de budget.

Mots-clés: production intégrée, développement rural, des aides agricoles.

\section{INTRODUCCIÓN}

De acuerdo con el Reglamento (CE) 1698/2005, relativo a la ayuda al desarrollo rural a través del Fondo Europeo Agrícola de Desarrollo Rural (FEADER) y con el marco competencial en España, cada Comunidad Autónoma ha elaborado un Programa de Desarrollo Rural para el periodo 2007-2013 en el que, además de las medidas horizontales y los elementos comunes, se incluyen medidas específicas para dar respuesta a las diferentes situaciones regionales y su gestión.

Los tres ámbitos de actuación comunitaria en desarrollo rural para el periodo 2007-2013 son los siguientes: 
1. La economía agroalimentaria y silvícola, que abarca el ciclo completo agrícola y forestal, desde la producción hasta la comercialización.

2. El medio ambiente, que se revela crecientemente como un factor económico decisivo en diversificar las economías rurales, además de contribuir a la conservación de la biodiversidad.

3. La economía y poblaciones rurales en un sentido amplio.

Estos ámbitos se articulan en torno a cuatro ejes:

- Eje 1: Aumento de la competitividad del sector agrícola y forestal.

- Eje 2: Mejora del medio ambiente y entorno rural.

- Eje 3: Calidad de vida en las zonas rurales y diversificación de la economía rural.

- Eje 4: LEADER.

El Programa de Desarrollo Rural de la Región de Murcia fue aprobado mediante la Decisión C (2008) 3838 de la Comisión Europea de 16 de julio de 2008 y modificado posteriormente mediante Decisión C (2009) 10318 de la Comisión de 15 de diciembre de 2009 por la que se aprobó la revisión del programa para el periodo de programación de 2007-2013. Este Programa contempla actuaciones en el Eje 2: Mejora del medio ambiente y del entorno rural y tiene como objetivos la mejora del medio ambiente rural, incidiendo positivamente en la recuperación y mantenimiento de la biodiversidad, la mejora la lucha contra la erosión, la implantación de técnicas de producción menos agresivas con el medio ambiente, el fomento de la superficie forestal como herramienta para la lucha contra la erosión, desertificación y el cambio climático, y finalmente la definición de actuaciones concretas sobre aquellos espacios naturales de mayor valor medioambiental. Con ello se busca garantizar el desarrollo sostenible de las zonas rurales, incentivando una gestión de las tierras compatible con la necesidad de preservar el medio ambiente y el paisaje natural, proteger y mejorar los recursos naturales. Las medidas propuestas para este eje son:

- Medida 211: Ayudas destinadas a compensar las dificultades naturales

- Medida 212: Zonas de montaña y ayudas a otras zonas con dificultades

- Medida 214: Ayudas agroambientales

- Medida 216: Inversiones no productivas

- Medida 221: Primera forestación de tierras agrícolas

- Medida 226: Medidas destinadas a la mitigación de la desertificación y prevención de incendios.

- Medida 227: Ayudas para inversiones no productivas.

En particular, la Medida 214: ayudas agroambientales, cofinanciada con fondos europeos, tiene como fin la continua integración de la actividad agraria en la sostenibilidad de los ecosistemas naturales, haciéndose necesario su constante incremento y adopción. Se promociona a los 
agricultores que suscriban de forma voluntaria compromisos agroambientales adicionales a los requeridos por la condicionalidad de la Política Agraria Común (PAC) con el fin de minimizar las posibles implicaciones medioambientales de la actividad agraria. Entre las líneas receptoras de estas ayudas se encuentra la de Producción Integrada, un sistema agrícola de obtención de vegetales que utiliza al máximo los recursos y los mecanismos de producción naturales y asegura a largo plazo una agricultura sostenible, introduciendo en ella métodos biológicos y químicos de control, amén de otras técnicas que compatibilicen las exigencias de la sociedad, la protección del medio ambiente y la productividad agrícola, así como las operaciones realizadas para la manipulación, envasado, transformación y etiquetado de productos vegetales acogidos al sistema (Real Decreto 1201/2002).

La región de Murcia se ha colocado a la cabeza en este tipo de manejo de cultivos, se ha adelantado a la normativa y la producción integrada es ya un hecho (VARÉS 2010). En este trabajo se estudia la aplicación y repercusión medioambiental de la ayuda agroambiental destinada a producción integrada incluida en el Eje 2 del Programa de Desarrollo Rural de la Región de Murcia 2007-2013, ayuda que nace en aras de compatibilizar la actividad agraria con la conservación del medio ambiente.

\section{LA PRODUCCIÓN INTEGRADA EN EL PROGRAMA DE DES- ARROLLO RURAL DE LA REGIÓN DE MURCIA.}

La Producción Integrada, a diferencia de la agricultura ecológica, no está regulada mediante reglamento europeo y hay normas técnicas de producción integrada a nivel nacional y autonómico. Su aplicación implica un mayor respeto al equilibrio de los ecosistemas, reduce la aplicación de tratamientos fitosanitarios reduciendo la contaminación en el aire, el agua y el suelo, y permite que los productos agrícolas tengan la menor cantidad posible de residuos químicos, mejorando la calidad de los mismos (Fig. 1).

Según datos del anuario de estadística 2009, publicado por el Ministerio de Medio Ambiente y Medio Rural y Marino, la superficie de Producción Integrada en la Región de Murcia ha experimentado un considerable aumento en el periodo 2008-2009, pasando de 12.534 ha a 23.753 ha, respectivamente (Fig. 2) siendo los cítricos el cultivo destinado a la mayor superficie 6.219 ha, seguida de los frutales con 5.346 hectáreas y frutos secos 4.845 ha. Los cultivos con menor superficie son los frutales de pepita con 193 ha (Fig. 3). 


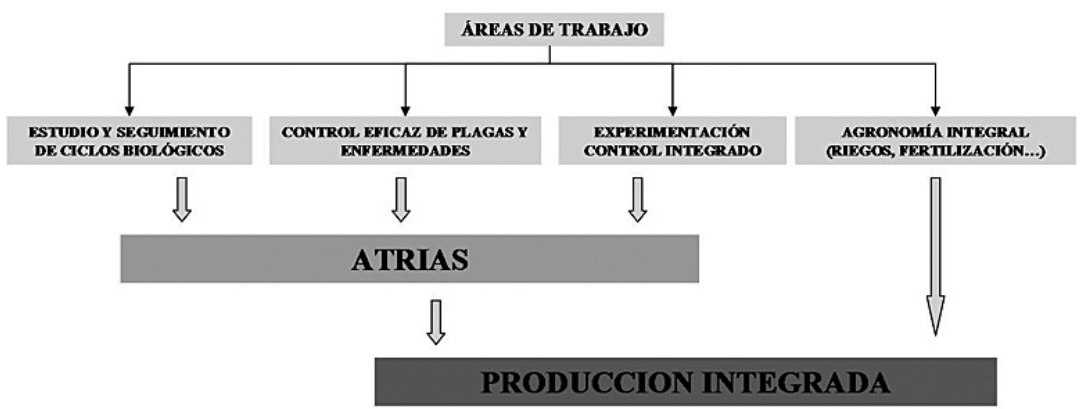

Figura 1: Esquema del sistema de producción integrada

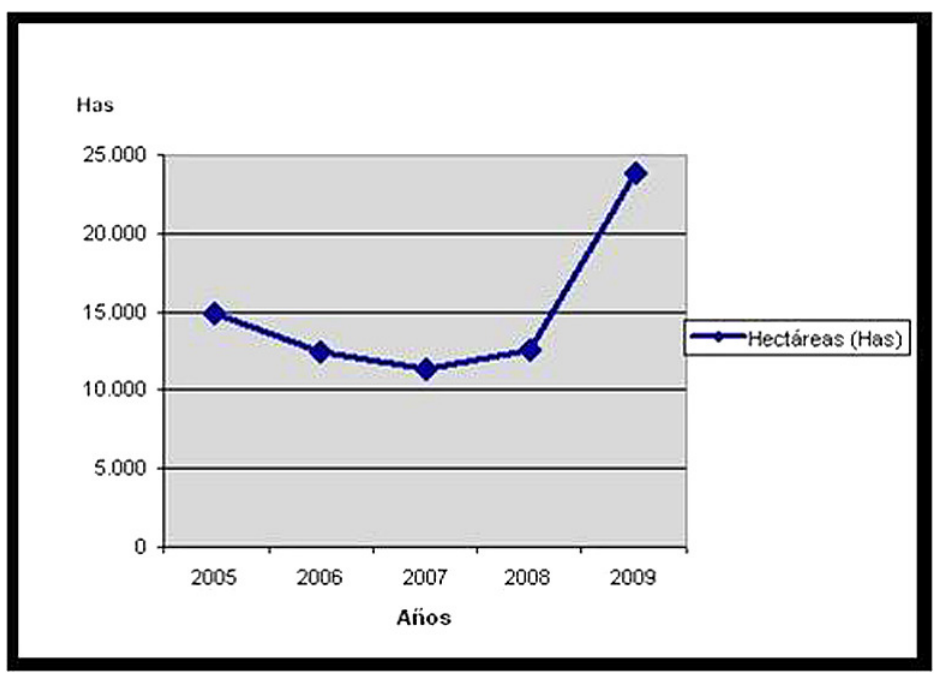

Figura 2: Evolución de la superficie de Producción Integrada en la Región de Murcia. Fuente: Elaboración propia a partir de datos publicados en MARM (2009).

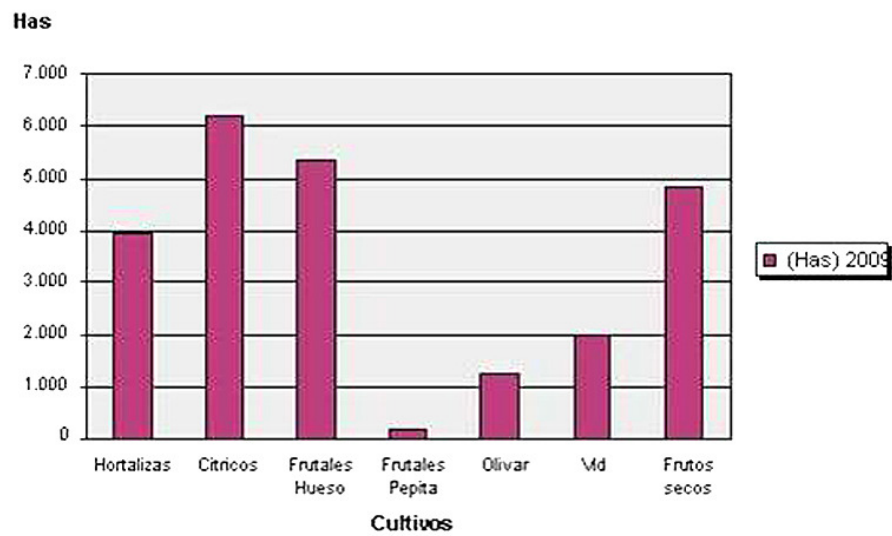

Figura 3: Superficies por cultivo en Producción Integrada en la Región de Murcia. Fuente: Elaboración propia a partir de datos publicados en MARM (2009). 
Los requerimientos a cumplir por las solicitudes son los siguientes:

\section{a) Objetivos:}

- Reducción de los tratamientos químicos.

- Conseguir un mayor respeto al equilibrio de los ecosistemas.

- Incrementar la materia orgánica del suelo.

- Obtener productos agrícolas que tengan la menor cantidad posible de residuos químicos.

\section{b) Requisitos de los beneficiarios:}

- Deben ser titulares de explotaciones agrícolas de la Región de Murcia, que se comprometan, por un período de cinco años, a cumplir los compromisos establecidos en las normas técnicas de producción integrada de la Región de Murcia que para el cultivo en cuestión estén establecidas en la Normativa nacional y autonómica).

- No pueden concederse la ayuda para aquellos cultivos para los que no existan normas técnicas de producción integrada en la Región de Murcia.

- Deben estar inscritos o haber solicitado la inscripción en el Registro de Productores y Operadores de Producción Integrada de la Región de Murcia.

\section{c) Requisitos de la explotación:}

- En los recintos SIGPAC objeto de ayuda se debe realizar el cultivo siguiendo las normas de Producción Integrada de la Región de Murcia.

- Los cultivos subvencionables son los que se relacionan en la tabla 1, ya que disponen de normas técnicas de producción integrada.

\begin{tabular}{|c|c|}
\hline \multicolumn{2}{|c|}{ CULTIVOS SUBVENCIONABLES } \\
\hline Apio & Almendro de secano \\
\hline Bróculi, coliflor y coles & Frutal de Pepita - Peral \\
\hline Cítricos & Olivo \\
\hline Escarola & Pimiento para Pimentón \\
\hline Lechuga & Pimiento invernadero \\
\hline Melocotonero, albaricoquero, ciruelo, cerezo y nectarino & Tomate \\
\hline Melón y sandia & Uva de Mesa \\
\hline Almendro de Regadío & Viña \\
\hline
\end{tabular}

Tabla 1: Cultivos subvencionables en Producción Integrada

Fuente: BORM no 225 (2008)

\section{d) Primas:}

Las primas para los productos producidos bajo las normas de producción integrada se indican en la tabla 2 y no varían durante los cinco años del compromiso. 


\begin{tabular}{|c|c|}
\hline CULTIVOS & PRIMA (C/ha/AÑO) \\
\hline $\begin{array}{c}\text { Frutales de hueso (albaricoquero, ciruelo, } \\
\text { melocotonero y nectarino) }\end{array}$ & 500 \\
\hline Frutales de pepita (peral) & 500 \\
\hline Cítricos & 500 \\
\hline Almendro & 150 \\
\hline Olivar & 280 \\
\hline Uva de mesa & 500 \\
\hline Uva vinificación & 250 \\
\hline Hortalizas aire libre o invernadero & 500 \\
\hline
\end{tabular}

Tabla 2: Primas ( $€ /$ ha/año) en Producción Integrada Fuente: BORM no 225 (2008)

\section{e) Descripción de los compromisos:}

Los compromisos que deben cumplirse durante el periodo de cinco años son:

- Producir bajo las normas técnicas de Producción Integrada de la Región de Murcia de acuerdo con las normas generales de producción control y certificación vigentes a nivel nacional y regional y a las modificaciones que de unas u otras pudieran producirse en el futuro.

- Disponer de una relación contractual con una Entidad de Control y Certificación autorizada.

- Comercializar la producción bajo la marca Producción Integrada.

- Contar con dirección técnica en la explotación, que asesore y elabore una memoria técnica en la que describa las actuaciones de la explotación.

\section{RESULTADO DE LA REPERCUSIÓN DE LAS AYUDAS AGRO- AMBIENTALES A LA PRODUCCION INTEGRADA.}

En el presente trabajo se han analizado los datos relativos a las Resoluciones Definitivas de las concesiones de ayuda a la producción integrada, publicados en los BORM no 223 de 26 de septiembre de 2009 y BORM no 252 de 31 de octubre de 2009. En cuanto al importe económico convocado se diferencian dos líneas de ayuda: línea 7020: cultivo del viñedo y línea 7001: resto de cultivos, siendo evaluadas las solicitudes por separado y haciendo la salvedad de que un mismo beneficiario puede tener concedidas más de una solicitud.

En la línea 7020: cultivo del viñedo, de diez solicitudes presentadas sólo una fue denegada. La superficie total concedida son 524,37 ha., lo que supone, con una prima de $250 € /$ ha/año, un importe total durante los cinco años de compromiso de 655.462,50 € (Tabla 3). 


\begin{tabular}{|c|c|c|c|c|c|}
\hline & $\begin{array}{c}\text { BENEFICIARIOS } \\
\text { CON CONCESIÓN }\end{array}$ & $\begin{array}{c}\text { SUPERFICIE } \\
\text { SOLICITADA } \\
\text { (ha) }\end{array}$ & $\begin{array}{c}\text { SUPERFICIE } \\
\text { CONCEDIDA } \\
\text { (ha) }\end{array}$ & $\begin{array}{c}\text { IMPORTE } \\
\text { CONCEDIDO } \\
\text { (c) }\end{array}$ & $\begin{array}{c}\text { IMPORTE } \\
\text { CONCEDIDO } \\
\text { \% }\end{array}$ \\
\hline $\begin{array}{c}\text { PERSONAS } \\
\text { FÍSICAS }\end{array}$ & 7 & 112,91 & 110,32 & $137.900,00$ & 21,04 \\
\hline HOMBRES & 5 & 97,10 & 95,46 & $119.325,00$ & 18,20 \\
\hline MUJERES & 2 & 15,81 & 14,86 & $18.575,00$ & 2,83 \\
\hline EMPRESAS & 2 & 420,90 & 414,05 & $517.562,50$ & 78,97 \\
\hline TOTAL & 9 & 533,81 & 524,37 & $655.462,50$ & 100 \\
\hline
\end{tabular}

Tabla 3: Concesión de la ayuda en la línea 7020: cultivo del viñedo Fuente: Elaboración propia a partir de datos publicados en BORM No 223 (2009)

En la línea 7001: resto de cultivos, se presentaron 637 expedientes y recibieron la concesión $333(52,28 \%)$. Un elevado número de solicitudes (304), pese a cumplir con todos los compromisos, no recibieron ayudas al no haber crédito suficiente para todas, ya que el importe total convocado era inferior al total solicitado y fue decisiva la concurrencia competitiva, aplicándose los criterios de prelación en la evaluación de las solicitudes. La superficie concedida es de 5.321,63 ha, ascendiendo a 7.995.273,50 $€$ el importe concedido durante los cinco años de compromiso.

El mayor número de beneficiarios en esta línea, son personas físicas, predominando los hombres sobre las mujeres. Las personas jurídicas representan un $11,41 \%$, muy inferior a las mujeres que representan el $22,52 \%$, sin embargo, ambos tienen una superficie total concedida similar, en torno a las 1.000 ha (Tabla 4). Esto es debido al menor tamaño de las explotaciones en manos de personas físicas, siendo la superficie media solicitada por las mujeres de 14 ha y por las empresas de 28 ha.

De gran interés es conocer la dimensión de las explotaciones con concesión (Tabla 5). En la línea 7001: resto de cultivos, los beneficiarios que han obtenido ayuda en una superficie menor a 10 ha son 170 para un total de 868,46 ha, destacando que sólo 11 beneficiarios, con superficies comprendidas entre 50 y 100 ha, han sumado una superficie total de 761,66 ha, siendo el importe total en ambos rangos de superficies similares. Esto pone de manifiesto que la producción integrada es solicitada por pequeños agricultores, siendo un incentivo para sus explotaciones. En la línea 7020: cultivo del viñedo, la mayor superficie e importes concedidos corresponden a un único expediente, una sociedad limitada, con una superficie de 341,28 ha.

\begin{tabular}{|c|c|c|c|c|c|}
\hline & $\begin{array}{c}\text { BENEFICIARIOS } \\
\text { CON CONCESIÓN }\end{array}$ & $\begin{array}{c}\text { SUPERFICIE } \\
\text { SOLICITADA } \\
\text { (ha) }\end{array}$ & $\begin{array}{c}\text { SUPERFICIE } \\
\text { CONCEDIDA } \\
(\mathrm{ha})\end{array}$ & $\begin{array}{c}\text { IMPORTE } \\
\text { CONCEDIDO } \\
(€)\end{array}$ & $\begin{array}{c}\text { IMPORTE } \\
\text { CONCEDIDO } \\
\%\end{array}$ \\
\hline $\begin{array}{c}\text { PERSONAS } \\
\text { FÍSICAS }\end{array}$ & 295 & $4.471,02$ & $4.248,88$ & $5.893 .112,50$ & 73,71 \\
\hline HOMBRES & 220 & $3.402,88$ & $3.216,60$ & $4.591 .262,00$ & 57,43 \\
\hline MUJERES & 75 & $1.068,14$ & $1.032,28$ & $1.301 .850,50$ & 16,28 \\
\hline EMPRESAS & 38 & $1.257,84$ & $1.072,75$ & $2.102 .161,00$ & 26,29 \\
\hline TOTAL & 333 & $5.728,86$ & $5.321,63$ & $7.995 .273,50$ & 100 \\
\hline
\end{tabular}

Tabla 4: Concesión de la ayuda en la línea 7001: resto de cultivos Fuente: Elaboración propia a partir de datos publicados en BORM No 252 (2009) 


\begin{tabular}{|c|c|c|c|c|c|}
\hline CóDIGo & $\begin{array}{l}\text { LINEA DE } \\
\text { AYUDA }\end{array}$ & $\begin{array}{c}\text { RANGO } \\
\text { SUPERFICIE } \\
\text { CONCEDIDA }\end{array}$ & $\begin{array}{c}\text { No TOTAL } \\
\text { BENEFICIARIOS }\end{array}$ & $\begin{array}{c}\text { TOTAL } \\
\text { SUPERFICIE } \\
\text { (ha) }\end{array}$ & $\begin{array}{l}\text { IMPORTE } \\
\text { (C) }\end{array}$ \\
\hline 7001 & $\begin{array}{l}\text { resto de } \\
\text { cultivos }\end{array}$ & $\begin{array}{c}0 \text { a } 10 \text { ha } \\
>10<50 \text { ha }> \\
50<100 \text { ha }> \\
100 \text { ha }\end{array}$ & $\begin{array}{c}170 \\
147 \\
11 \\
5\end{array}$ & $\begin{array}{c}868,46 \\
3.077,80 \\
761,66 \\
613,71\end{array}$ & $\begin{array}{c}1.595 .788,00 \\
4.448 .178,50 \\
1.439 .175,00 \\
512.132,00\end{array}$ \\
\hline 7020 & $\begin{array}{l}\text { cultivo del } \\
\text { viñedo }\end{array}$ & $\begin{array}{c}0 \text { a } 10 \mathrm{ha} \\
>10<50 \mathrm{ha} \\
>50<100 \mathrm{ha} \\
>100 \mathrm{ha}\end{array}$ & $\begin{array}{l}3 \\
4 \\
1 \\
1\end{array}$ & $\begin{array}{c}20,96 \\
89,36 \\
72,77 \\
341,28\end{array}$ & $\begin{array}{c}26.200,00 \\
111.700,00 \\
90.962,50 \\
426.600,00\end{array}$ \\
\hline
\end{tabular}

Tabla 5: Beneficiarios por línea de ayuda y según tamaño de superficie concedida Fuente: Elaboración propia a partir de datos publicados en BORM No 223 (2009) y BORM 252 (2009)

El cultivo con mayor superficie concedida fue el almendro, seguido de los cultivos intensivos (frutales, uva de mesa, cítricos y hortalizas), viñedo y olivar (Fig. 4). El cultivo del almendro obtuvo las mayores puntuaciones en el proceso de evaluación de solicitudes porque es un cultivo extensivo que se encuentra en zonas desfavorecidas y reúne la mayoría de los criterios de prelación.

La Producción Integrada, en conjunto, se ha visto perjudicada al hacer separación de líneas en función del cultivo, ya que la línea línea 7020: cultivo del viñedo, solamente demandó un 9,34\% del importe de la convocatoria, por el contrario, la línea 7001: resto de cultivos tuvo una mayor demanda, produciéndose un déficit total de 18.638.952 € (Tabla 6).

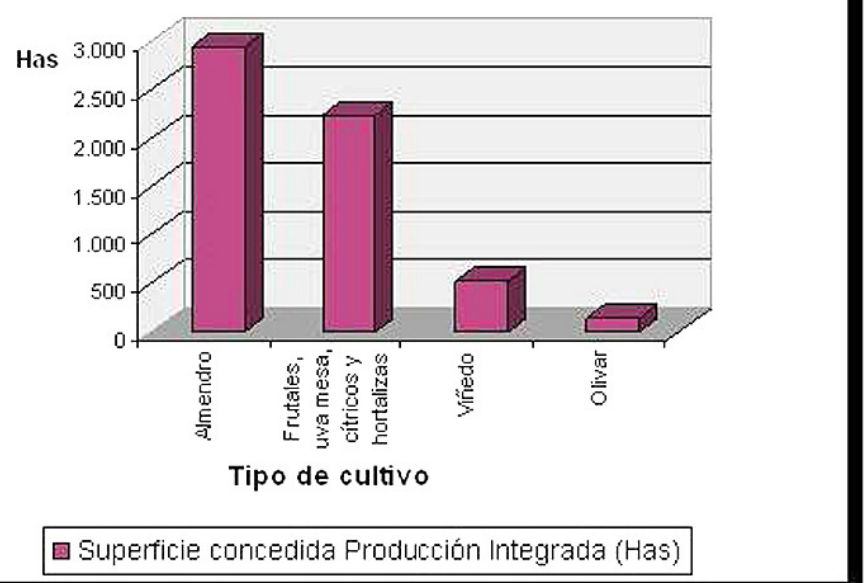

Figura 4: Superficie concedida según el tipo de cultivo.

Fuente: Elaboración propia a partir de datos publicados en BORM No 223 (2009) y BORM 252 (2009). 


\begin{tabular}{|c|c|c|c|c|c|}
\hline \multirow[b]{2}{*}{ CódIGO } & \multirow[b]{2}{*}{$\begin{array}{l}\text { LINEA DE } \\
\text { AYUDA }\end{array}$} & \multirow[b]{2}{*}{$\begin{array}{c}\text { CONVOCATORIA } \\
\text { IMPORTE } \\
\text { (C) }\end{array}$} & \multicolumn{2}{|c|}{ CONCESIÓN } & \multirow[b]{2}{*}{$\begin{array}{c}\text { IMPORTE NO } \\
\text { CONCEDIDO } \\
\text { (E) }\end{array}$} \\
\hline & & & $\begin{array}{l}\text { IMPORTE } \\
\text { (C) }\end{array}$ & $\begin{array}{c}\text { CONCEDIDO } \\
\text { RESPECTO AL } \\
\text { CONVOCADO } \\
(\%)\end{array}$ & \\
\hline 7001 & resto de cultivos & $8.015 .841,85$ & $7.995 .273,50$ & 100 & $18.638 .952,50$ \\
\hline 7020 & cultivo del viñedo & $7.018 .110,50$ & $655.462,50$ & 9,34 & -- \\
\hline & TOTAL & $15.033 .952,35$ & $8.650 .736,00$ & & $18.638 .952,50$ \\
\hline
\end{tabular}

Tabla 6: Importes económicos concedidos en Producción Integrada

Fuente: Elaboración propia a partir de datos publicados en BORM No 223 (2009) y BORM 252 (2009)

A pesar de que para la línea línea 7020: cultivo del viñedo se diera una diferencia de 6.362.648 $€$ entre el importe total convocado (7.018.110,50 $€)$ y el importe total concedido $(655.462,50 €)$, no pudo destinarse esta cantidad a la línea 7001 : resto de cultivos para compensar la falta de crédito, porque la orden de convocatoria (Orden de 22 de septiembre de 2008), establecía en su artículo 4.4 que cuando se comprobase que en alguna de las líneas existe remanente se destinará a las líneas en las que queden solicitudes pendientes de concesión conforme al siguiente orden:

a) Agricultura ecológica.

b) Conservación de suelos agrícolas (lucha contra la erosión).

De este modo, la línea 7001: resto de cultivos se ha visto perjudicada, en este caso por insuficiencia de crédito, ya que 8.733 ha quedaron sin ayuda, pertenecientes mayoritariamente a empresas, lo que hubiera supuesto un importe total de 16.047.505 $€$ que sumado a las solicitudes no concedidas por personas físicas asciende a un total de 18.638.952,50 $€$, el importe no concedido (Tabla 7).

\begin{tabular}{|c|c|c|c|c|c|}
\hline & $\begin{array}{c}\text { BENEFICIARIOS } \\
\text { SIN CONCESIÓN }\end{array}$ & $\begin{array}{c}\text { SUPERFICIE } \\
\text { SOLICITADA } \\
\text { (ha) }\end{array}$ & $\begin{array}{c}\text { SUPERFICIE } \\
\text { NO } \\
\text { CONCEDIDA } \\
\text { (ha) }\end{array}$ & $\begin{array}{c}\text { IMPORTE NO } \\
\text { CONCEDIDO } \\
\text { (C) }\end{array}$ & $\begin{array}{c}\text { IMPORTE NO } \\
\text { CONCEDIDO } \\
\text { \% }\end{array}$ \\
\hline $\begin{array}{c}\text { PERSONAS } \\
\text { FÍSICAS }\end{array}$ & 147 & $1.812,33$ & $1.698,55$ & $2.591 .447,00$ & 13,90 \\
\hline HOMBRES & 104 & $1.072,13$ & 978,24 & $1.750 .209,00$ & 9,39 \\
\hline MUJERES & 43 & 740,20 & 720,31 & $841.157,00$ & 4,51 \\
\hline EMPRESAS & 104 & $8.404,13$ & $7.035,15$ & $16.047 .505,50$ & 86,10 \\
\hline TOTAL & 251 & $10.216,46$ & $8.733,70$ & $18.638 .952,50$ & 100 \\
\hline
\end{tabular}

Tabla 7: Solicitudes no concedidas por insuficiencia de crédito en la línea 7001: resto de cultivos

Fuente: Elaboración propia a partir de datos publicados en BORM No 252 (2009)

Respecto a los cultivos que no obtuvieron concesión, frutales, uva de mesa, cítricos y hortalizas, fueron los más perjudicados en el proceso de evaluación de solicitudes quedando una gran superficie sin conceder (6.626 ha) (Figura 5). El carácter intensivo de las explotaciones y su ubicación hace que no reúnan los criterios de prelación de mayor puntuación.

En la tabla 8 se muestra la evaluación de los 339 beneficiarios (tres solicitudes tienen solicitadas las dos líneas) que obtuvieron ayudas de 
Producción Integrada en las dos líneas siguiendo los criterios establecidos en el proceso de concesión. Se observa que la mayor parte tienen su explotación agraria en zonas desfavorecidas (zonas de montaña o de despoblamiento) y que el $62,5 \%$ son agricultores a título principal, lo que refleja que esta ayuda va destinada mayoritariamente a profesionales de la agricultura. Un $9,73 \%$ de los beneficiarios cumplen el criterio de disponer más de $50 \%$ de su explotación dentro de la Red Natura 2000 y el 78,17\% de los beneficiarios no han recibido esta ayuda en el anterior programa 2000-2006.

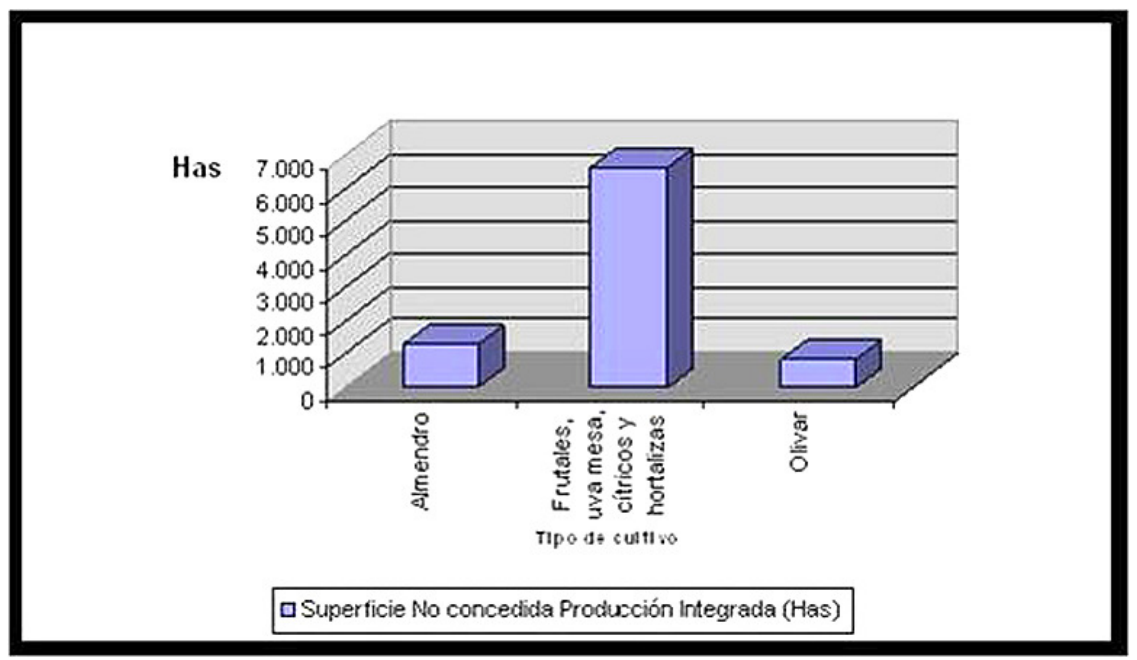

Figura 5: Superficie no concedida en producción integrada (7001) según el tipo de cultivo (ha).

Fuente: Elaboración propia a partir de datos publicados en BORM No 252 (2009).

\begin{tabular}{|c|c|c|c|}
\hline CRITERIOS DE CONCESIÓN & PUNTOS & No SOLICITUDES* & $\%$ \\
\hline $\begin{array}{l}\text { a) Explotaciones cuya superficie se encuentre en más } \\
\text { de un } 50 \% \text { dentro de la Red Natura } 2000 \text { recogidas } \\
\text { en el ANEXO III, siempre y cuando las actuaciones } \\
\text { objeto de la ayuda sean compatibles y adecuadas a } \\
\text { los objetivos de conservación del lugar, establecidos } \\
\text { por el órgano competente de la gestión de la Red } \\
\text { Natura } 2000, \text { y, en su caso, de aquellos previstos en } \\
\text { los Planes de Gestión correspondientes. }\end{array}$ & 32 & 33 & 9,73 \\
\hline b) Explotaciones calificadas como prioritarias. & 16 & 23 & 6,78 \\
\hline c) Explotaciones cuyo titular tenga la condición de ATP. & 8 & 212 & 62,54 \\
\hline $\begin{array}{l}\text { d) Explotaciones cuya superficie elegible para la } \\
\text { concesión de la ayuda se encuentre, en más del } \\
50 \% \text {, dentro de alguna de las zonas desfavorecidas } \\
\text { establecidas y sean susceptibles de percibir la } \\
\text { indemnización compensatoria. }\end{array}$ & 4 & 161 & 47,49 \\
\hline $\begin{array}{l}\text { e) Explotaciones que no se beneficiaron de la misma } \\
\text { línea de ayuda solicitada en el anterior Programa de } \\
\text { Desarrollo Rural } 2000-2006 \text {. }\end{array}$ & 2 & 265 & 78,17 \\
\hline
\end{tabular}

Tabla 8: Evaluación de las solicitudes que cumplen los criterios de prelación Fuente: Elaboración propia a partir de datos publicados en BORM No 223 (2009) y BORM 252 (2009)

*Una misma solicitud puede cumplir varios criterios de concesión. 
La línea 7001: resto de cultivos, debido a la insuficiencia de crédito ya comentada, se sometió a concurrencia competitiva, siguiendo los criterios de prelación que se señalan en la tabla 9.

\begin{tabular}{|c|c|c|c|}
\hline CRITERIOS CUMPLIDOS & PUNTUACIÓN & BENEFICIARIOS & CONCESIÓN \\
\hline a), b), c), d), y e) & 62 & 0 & \multirow{23}{*}{ SI } \\
\hline a), b), c), y d) & 60 & 1 & \\
\hline a) b) y c) & 56 & 0 & \\
\hline a), c), d) y e) & 46 & 0 & \\
\hline a), c) y d) & 44 & 0 & \\
\hline a), c) y e) & 42 & 3 & \\
\hline a) y c) & 40 & 2 & \\
\hline a), d) y e) & 38 & 11 & \\
\hline a) y d) & 36 & 6 & \\
\hline a) y e) & 34 & 7 & \\
\hline a) & 32 & 3 & \\
\hline b), c), d), y e) & 30 & 9 & \\
\hline b), c) y d) & 28 & 4 & \\
\hline b), c) y e) & 26 & 3 & \\
\hline b) y c) & 24 & 1 & \\
\hline b), d) y e) & 22 & 0 & \\
\hline b) y e) & 18 & 1 & \\
\hline b) & 16 & 4 & \\
\hline c), d), y e) & 14 & 22 & \\
\hline c) y d) & 12 & 11 & \\
\hline c) y e) & 10 & 112 & \\
\hline c) & 8 & 42 & \\
\hline d) y e) & 6 & 91 & \\
\hline d) y e) & 6 & 6 & \multirow{4}{*}{ NO } \\
\hline d) & 4 & 20 & \\
\hline e) & 2 & 165 & \\
\hline Ninguno & 0 & 60 & \\
\hline Total & 584 & & \\
\hline
\end{tabular}

Tabla 9: Puntuación obtenida en el proceso de evaluación de solicitudes de la línea 7001: resto de cultivos

Fuente: Elaboración propia a partir de datos publicados en BORM No 252 (2009)

De las 637 solicitudes presentadas, 584 cumplían con todos los requisitos, pero solamente obtuvieron concesión 333. La puntuación de corte fue 6 , que se corresponde a la suma de los criterios d) y e). Debido al empate en la puntuación, prevaleció la menor superficie solicitada que fue 73,03 has. Las solicitudes que tenían una puntuación inferior a 4 no obtuvieron concesión de la ayuda y las que sumaban 6 solamente se concedieron a las que tenían una superficie solicitada menor de 73,03 ha.

Como consecuencia del gran número de expedientes que cumplían con todos los compromisos y no obtuvieron concesión, en el año 2010 se publi- 
ca una nueva orden de convocatoria solamente para Producción Integrada, a través de la Orden de 29 de julio de 2010 de la Consejería de Agricultura y Agua, modificada por la Orden de 29 de julio de 2011, de la Consejería de Agricultura y Agua (BORM, no 179). Dicha convocatoria se encuentra pendiente de tramitación, en ella se retrasa a 2012 el año de inicio de los compromisos y, por consiguiente, a 2016 el de su finalización.

\section{CONCLUSIONES}

La ayuda agroambiental de Producción Integrada incluida en el Programa de Desarrollo Rural de la Región de Murcia ha tenido una importante aceptación en el sector agrario murciano, si bien no ha cubierto las expectativas de un importante número de agricultores que han visto desestimadas sus solicitudes por falta de presupuesto.

Las solicitudes concedidas en ambas líneas han sido 342, siendo los beneficiarios 339, ya que tres de ellos solicitaron las dos líneas de ayuda. El importe total concedido es de $8.650 .736 €$ y ha recaído, mayoritariamente, en personas físicas, 302 expedientes, frente a los 40 expedientes correspondientes a personas jurídicas.

La superficie total con derecho a ayuda en la línea 7001: resto de cultivos es de 14.055,33 ha, de las cuales solamente obtuvieron concesión $5.321,63$ ha, quedando $8.733,70$ ha sin conceder, a pesar de que cumplían con todos los compromisos, debido a la falta de crédito presupuestario en esta línea. El cultivo con mayor superficie concedida fue el almendro, al encontrarse en zonas desfavorecidas y reunir la mayoría de los criterios de prelación, seguido de los cultivos intensivos (frutales, uva de mesa, cítricos y hortalizas), viñedo y olivar. Respecto a los cultivos que no obtuvieron concesión, los frutales, uva de mesa, cítricos y hortalizas, fueron los más perjudicados en el proceso de evaluación de solicitudes, el carácter intensivo de las explotaciones y su ubicación hacen que no reúnan los criterios de prelación de mayor puntuación, quedando una gran superficie sin conceder, 6.626 ha.

La superficie total concedida en la línea 7020: cultivo del viñedo fue de 524,37 has, siendo la demanda muy inferior a la esperada en un principio, quedando un remanente de 6.362.648 $€$.

El mayor número de solicitudes y beneficiarios se encuentran en aquellas explotaciones cuyo titular tiene la condición de agricultor a tiempo parcial, se encuentran, en más del $50 \%$, en zonas desfavorecidas y no se beneficiaron de la misma línea de ayuda en el anterior Programa de Desarrollo Rural.

La línea de Producción Integrada fomenta una agricultura sostenible que realiza prácticas respetuosas con el medio ambiente, beneficiando tanto a la población del medio rural, como a su estructura económica, a los que se suman los beneficios de carácter ambiental, tales como: favo- 
recer la conservación de especies vegetales autóctonas, reducir el impacto sobre el medio hídrico y el suelo y conservar el paisaje.

\section{BIBLIOGRAFÍA}

BOLETÍN OFICIAL DE LA REGIÓN DE MURCIA. BORM, No 225 (2008): Orden de 22 de septiembre de 2008, de la Consejería de Agricultura y Agua por la que se establecen en la Región de Murcia, las bases reguladoras y se aprueba la convocatoria correspondiente al año 2008 de las líneas de ayuda 7000, 7001, 7002, 7003, 7004, 7006, 7008, 7019 y 7020. BORM, No 223 (2009): Anuncio por el que se da publicidad a las propuestas de resolución definitiva de las ayudas 7000, 7002, 7003, 7004, 7006, 7008, 7019 y 7020.

BORM, No 252 (2009): Anuncio por el que se notifican las propuestas de resolución definitiva de las líneas de ayuda 7001: Producción integrada. 7008: Conservación de variedades vegetales en peligro de extinción.

BORM, No 177 (2010): Orden de 29 de julio de 2010 de la Consejería de Agricultura y Agua, por la que se establecen, en la Región de Murcia, las bases reguladoras de las líneas de ayuda y se aprueba la convocatoria correspondiente al año 2010 para la línea de Producción Integrada.

BORM, No 179 (2011): Orden de 29 de julio de 2011, de la Consejería de Agricultura y Agua, por la que se modifica la convocatoria correspondiente al año 2010 de la línea de ayudas para la producción integrada, aprobada mediante la Orden de 29 de julio de 2010 de la Consejería de Agricultura y Agua.

CONSEJERÍA DE AGRICULTURA Y AGUA DE LA REGIÓN DE MURCIA (2007): Programa de Desarrollo Rural de la Región de Murcia 2007-2013. En http://www.carm.es

JIMÉNEZ DÍAZ, R. M. (1998): "Concepto de sostenibilidad en Agricultura". En Agricultura Sostenible (JÍMENEZ DÍAZ R.M. Y LAMO DE ESPINOSA, J., COORDS.). Mundi- Prensa, Madrid: 3-13 pp.

LABRADOR, J. Y ALTIERI. M. A. (2001): Agroecología y Desarrollo Rural. Mundi-Prensa. Universidad de Extremadura.

MARM (2009a): Anuario de estadística 2009. Ministerio de Medio Ambiente y Medio Rural y Marino. En http://www.marm.es/es/

MARM (2009b): Plan estratégico nacional de desarrollo rural 2007-2013. Ministerio de Medio Ambiente y Medio Rural y Marino. En http://www.marm.es/es/

VARÉS, F. (2010): "La producción integrada, una realidad en nuestra agricultura". Vida Rural, 306. En http://www.vidarural.es 